\title{
A Critical Analysis of Biomedical Image Classification on Deep Learning
}

\author{
${ }^{1}$ Rose Lu and ${ }^{2}$ Dawei Pan \\ ${ }^{1,2}$ School of Automation, Tongji University, Shanghai, China, 200070 \\ 1'daweipanchina@gmail.com
}

\begin{abstract}
Article Info
Journal of Biomedical and Sustainable Healthcare Applications (http://anapub.co.ke/journals/jbsha/jbsha.html) Doi: https://doi.org/10.53759/0088/JBSHA202101014
\end{abstract}

Received 25 October 2020; Revised form 30 December 2020; Accepted 25 April 2021.

Available online 05 July 2021.

(C2021 Published by AnaPub Publications.

\begin{abstract}
In computer-aided diagnostic technologies, deep convolutional neural image compression classifications are a crucial method. Conventional methods rely primarily on form, colouring, or feature descriptors, and also their configurations, the majority of which would be problem-specific that has been depicted to be supplementary in image data, resulting in a framework that cannot symbolize high problem entities and has poor prototype generalization capability. Emerging Deep Learning (DL) techniques have made it possible to build an end-to-end model, which could potentially general the last detection framework from the raw clinical image dataset. DL methods, on the other hand, suffer from the high computing constraints and costs in analytical modelling and streams owing to the increased mode of accuracy of clinical images and minimal sizes of data. To effectively mitigate these concerns, we provide a techniques and paradigm for DL that blends high-level characteristics generated from a deep network with some classical features in this research. The following stages are involved in constructing the suggested model: Firstly, we supervisedly train a DL model as a coding system, and as a consequence, it could convert raw pixels of medical images into feature extraction, which possibly reflect high-level ideologies for image categorization. Secondly, using image data background information, we derive a collection of conventional characteristics. Lastly, to combine the multiple feature groups produced during the first and second phases, we develop an appropriate method based on deep neural networks. Reference medical imaging datasets are used to assess the suggested method. We get total categorization reliability of 90.1 percent and 90.2 percent, which is greater than existing effective approaches.
\end{abstract}

Keywords - Deep Learning (DL), Coding Network with Multilayer Perceptron (CNMP), Support Vector Machine (SVM), Convolutional Neural Networks (CNNs)

\section{INTRODUCTION}

Amongst the most basic jobs in computational intelligence is image segmentation, which involves assigning one or more descriptors to an image. Mid-level or Low-level features are retrieved to characterize the picture in classical image analysis, and then an adaptable classifier is employed to provide labels. Convolutional Neural Networks (CNNs) [1] high image representation has outperformed hand-crafted low-level and mid-level components in recent times. Both information extraction systems are merged in the convolution neural network, which is trained from start to finish. Biomedical image analysis and computer-aided diagnostics have both benefitted from Deep Learning (DL) approaches. With the fast advancement of computerized image capturing and storage technology, picture interpretation by computing software has become a popular and active issue in computer vision and application-specific research. Rapid and precise labeling or rating of biomedical images has become a vital approach in most medical professions in order to develop smart computer-aided diagnosis systems. Every year in the U.S., for instance, a large number of individuals are treated with skin malignancy. Numerous lives could be saved if the disease was diagnosed early.

In the context of biomedical image analysis, numerous research papers have been published. The concentrating region, contrasts, and white balance of image data collected from different sources, nevertheless, may differ. Furthermore, imaging techniques frequently contain internal components with various textures and input image intensities. It would have been challenging to effectively categorize specific classes if we utilized only conventional model to detect clinical data. Machine learning has risen to prominence in recent years as one of the most exciting areas of studies in computer technology and software solutions. Many studies have endeavored to apply DL to non-medical images as a result of advancements in the field. The structure of the DL model was first addressed by Pladere et al. [2]. To fix image issues in the future in the future, a variation of deep schemes has been developed. In the ILSVRC-2010 (ImageNet Large-Scale Visual Recognition Challenge-2010), Yuan, Chiang, Tang, and Haro [3] trained a DL model to perform classification of images, accomplishing best-in-class results. The impact of deep configuration depth on machine vision efficiency was discussed by Kunickaya et al., [4]. This framework has already sparked a lot of interest in applying this novel technique to clinical computer vision problems, thanks to these productive research findings.

Biomedical image analysis [5] is among the most pressing issues in image processing, with the goal of categorizing medical pictures into distinct groups to aid clinicians in illness diagnosis and study. The categorization of medical images may be broken down into two parts. The first phase is to identify the image's useful elements. The characteristics are then 
used to create frameworks, which classify the image datasets in the second phase. Healthcare practitioners utilized to extract characteristics from medical photographs and categorize them into distinct groups using their professional knowledge, which was a tough, time-consuming and tedious tasks. This technique is protected at impacting non-repeatable or inconsistency results. Biomedical image analysis applications study has a lot of potential, based on previous research. The efforts of researchers have resulted in a huge number of publications in this field. Nonetheless, we seem to be unable to complete this objective effectively at this time. If we can complete the categorization process well, the data will aid medical professionals in diagnosing disorders that need further investigation. As a result, it's critical to figure out how to do this activity properly.

Prior to the appearance of deep network [6], a great number of earlier researches employed shallow architectures for therapeutic image recognition, which depended mostly on form, colour, and texture data, and also their permutations. The fundamental issue with all of these systems is that the recovered components are generally alluded to as low-level characteristics, which lack representations capacity for high-level domain knowledge ideas and have weak generalization capability. Deep network, on the other hand, have had a great deal of successes in the non-medical image industry. DL-based approaches, that are the most fascinating ensemble learning algorithm, offer an efficient means to develop end-to-end framework, which typically generates categorization process class from raw biomedical image pixel. Since architectures need big datasets to get remarkable properties, deep modelling solutions in the therapeutic image analytics area require a lot of work to cope up with the other fields of imagery.

Clinical images [7], on the other hand, are notoriously difficult to get, hence medical databases are frequently limited. As a result, if we employ a DL model to solve a problem with a little dataset, we are likely to overfit the models. Aside from these issues, the model's generalisability has been demonstrated to be inferior, and deep neural prototype typically necessitates a significant computation. We provide a unique revolutionary deep model that blends conventional and deep characteristics to address these issues regarding traditional approaches vs deep models. This approach can use ensemble techniques to autonomously retrieve high-level characteristics for identifying medical pictures, in addition to using current clinicians' expertise.

In this work, we will evaluate the Coding Network with Multilayer Perceptron (CNMP) approach for learning multiresolution properties, which blends DL model with standard picture characteristics. We aim to assist physicians save time and effort by accurately detecting photographs, thus we're using this technology to categorize medical images. Furthermore, it's worth noting that one of the most important aspects of our technique is retrieving characteristics from the query image, which the deep prototype can do instantaneously while existing algorithms can do manually. This method may employ both low-level and high-level representations of an image at the same time while avoiding the usage of single representations or attribute. It can also condense two kinds of attributes automatically, eliminating the need for timeconsuming model validation.

Medical picture categorization has at least two difficulties: (a) what characteristics can we retrieve from a tiny clinical picture collection that is efficacious? Generally, medical picture collections are so tiny that extracting discriminant information is impossible. Even though the suggested technique can achieve excellent classification performance regardless of the quantity of the picture collection, its real application usefulness is severely restricted. A novel data augmentation strategy is provided in Lafarge and Koelzer in [5] to prevent the acquisition of nonvalid attributes while working with limited datasets. Subsequently, to improve their model's performance, they employed an extended dataset. Finding a technique that can generate discriminative characteristics from a short dataset is thus important. (b) How can various sorts of characteristics from different models be merged fast and efficiently? The concept of directly integrating feature matrices into a bigger feature vector and finding a single percentage variable between multiple characteristics seems to be simple to express. This strategy, nonetheless, usually necessitates trial and error to retrain the variables and cannot provide a superior result. We could get greater precision than these approaches if we could create a more favourable fusion methodology. As a result, there's a lot of pressure to combine the features successfully.

The following are the primary contributions of this article:

- To categorize medical pictures, we suggested a deep framework that incorporates both high-level and conventional characteristics. Rather than employing domain-transferred convolution neural network, like the Domain-Transferred Convolutional Neural Networks (DTCNNs) projected Pang, Yu, and Orgun in [6], it overtly trained a deep Neural Networks (CNNs) [10] labeled the coding networks to actual high-levels attributes. The generalisability of the learning algorithm as well as the greatest performance might be enhanced by using typical medical picture elements.

- To combine high-level elements with conventional characteristics, we used two ways. One way is to allocate fixed argumentation representations of the assertion between high-level and conventional characteristics, in which the conventional approach is tedious, time-consuming, and impossible to implement. Another solution is given to address these difficulties: a new infrastructure that could not only integrate the elements together but also modify their ratios autonomously.

The remaining part of the paper has been organized as follows: Section II focusses on a review of the relevant literature texts. Section III analyses the proposed framework. Section IV provides a critical analysis of the paper. Lastly, Section V concludes the paper.

\section{LITERATURE REVIEW}

Many approaches have been presented to address these difficult picture categorization issues, which may be divided into two categories: standard techniques and deep modeling techniques. Colour and textures, regression trees, and Support Vector 
Machine (SVM), as reviewed by Son and Kim [11], are examples of traditional approaches. DL models have been used to categorize medical pictures in a number of studies. In this part, we'll go through the prior work on picture categorization in depth. After that, a study of the literature on data augmentation for image categorization problems will be conducted.

Reiter et al. in [12] have developed two methods that use texture and color cues to identify melanomas in dermoscopy pictures. To categorize lesions, one approach utilizes global characteristics while the other utilizes feature points. On a 176 sample of mammogram images from the clinical Pedro Hispano, the findings were displayed. Beasley et al. [13] developed a form, color, and texture-based melanoma diagnostic method based on the Web. On 1200 dermoscopy pictures, this technique achieved a Specificity (SP) of 86 and Sensitization (SE) of 86. Pandiar et al. [14] used a blend of color - texture parameters to compare the regions of granular among melanoma and comparable regions in non-melanoma lesions. On a data with 88 malignant tumors and 200 non-melanoma tumors, their article employed the Receiver Operating Characteristics (ROC) curve to show the system's highest extraction efficiency. Chegraoui et al. [15] were the first to use new color and texture features descriptor based on area to detect cancer in photos. Texture characteristics in their models are founded on Gabor, and feature sets are obtained using homomorphic filtration, which may solve the issue of varied orientations, magnification, and lighting.

To aid in the diagnosis of Alzheimer 's illness, Abou et al. [16] recommended a random forest type based on Single Photon Emission Computed Tomography (SPECT) image segmentation. In order to organize the random forests, they first retrieved score characteristics from the picture datasets using partial least squares. The use of this method as a classifier aids in the classification of all of the photos. The particular technique is to categorize the picture to the nearest centroid recessively till achieving a single tree leaflet, which is the image categorization. This is why the most essential feature of this algorithm is that it can build on the prior model without having to retrain the pictures from start, a process known as "learning algorithms."

To categorize computed tomography brain pictures into diseased and healthy classifications, Rumack and Johnson [17] presented a classifier based on a partial Fourier series and a non - parallel SVMs. As a result, it was a dichotomous categorization exercise. The system extracted spectrum characteristics from a given picture using a weighted-type proportionate Fourier transform, then applied hierarchical clustering to minimize the dimension of the inferential data. Finally, the spectrum characteristics it included were input into SVMs. However, the dataset in this work, which consists of 90 T2-weighted MRI brain pictures, is somewhat tiny. Despite its impressive results, it is evident that it is not well suited to a bigger sample.

To categorize lung input images, Li, Zhan, Xu, Kwong, and Zhang [18] structured the patented Convolutional Neural Networks (CNNs). To avoid fitting problem, the network only had one convolution layers to retrieve feature representations, and it outperformed SIFT Descriptors, rotation-invariant LBP (Local Binary Pattern) [19] elements, and unsupervised deep features centred on RBM (Restricted Boltzmann Machine) as seen by Peng, Gao, and Li [20]. Nagesha, Mahesh, and Gowrishankar [21] presented a modified DL methodology referred to as the PCANet (Principal Component Analysis Network that was utilized by Rajesh and Chaturvedi [22] in integration with the spatial data patterns of colour photos to accomplish sophisticated classification accuracy in data sources.

Chen, Agarwal, and Nguyen [23] used an ImageNet-trained CNNs to detect distinct sorts of diseases in the chest x-ray clinical image. The authors obtained a greater type of precision by incorporating CNN elements with custom attributes. Bellon et al. [24] detailed why the transfer training could be fundamental when handling clinical images. They doublechecked their results of the thoracoabdominal Lymph Nodes (LN) identifications. Scattered transform, initially presented Zirka, Moroz, and Arturi [25], was utilized by Rampun et al. [26] to retrieve the features alongside Local Quinary Patterns (LQPs) and LBP for the treatment and diagnosis of lung cancer, which was considered to be resilient to minor deformations in biomedical imaging. The authors tested the two-dimensional Hela set of data and the Pap smear data for efficacy and performance. Avau, Chintinne, Baudry, and Buxant [27] account for DL approaches for robotically perceiving IDC (Invasive Ductal Carcinoma) tissue classification in WSI (Whole Slid Images) for cancer of the breast that has been validated using datasets of 162 patients treated and diagnosed with IDC and attained a balanced precision.

In order to effectively categorize X-ray images, [28] proposed an approach that incorporated DTCNNs with SSP (Sparse Spatial Pyramid). They utilized 19-layer CNNs (VGG-19) recommended by the authors as the transferred networks within this contribution that could possibly disregard biomedical image features. This approach, on the contrary, provided a fresh ideology on the condition. Authors presented a multi-scale high-level feature representation for the verification of faces according to the authors that they called DeepID (Deep Hidden Identity Features). Structures gotten from the $3^{\text {rd }}$ and $4^{\text {th }}$ CNNs layer are incorporated onto the multi-scale features. The researchers issued a logistic regression fusion approach for fusing shape and color data without being connected to any of them. To compensate for the flaws of not addressing the visual words' statistical dependencies, their system explicitly weighted them. Rahim and Manson [29] used KPCA (Kernel Principal Component Analysis) as fusion technique to potentially uncover non-linear correlation between retrieved color and texture dataset, and therefore be utilized the probabilistic methodology to automatically pick the best feature set from the fused data. Because they utilize Convolutional Neural Networks (CNNs) or conventional techniques to identify medical pictures, all of the following systems have flaws.

In [30], traditional approaches, regardless of whether attributes (moment colour or texture features) are utilized, do not enough in classifying medical photos purely on the basis of experience-based features. The transfer-learning networks find it relatively simple to disregard the peculiarities of medical pictures in deep models. Furthermore, the vast majority of medical picture classification research relies on binary categorization. In actuality, we are often required to complete a 
multiclass categorization problem. We describe a novel method to overcome these issues and boost the effectiveness of clinical image segmentation. Section III presents a review of the proposed framework in this contribution.

\section{PROPOSED FRAMEWORK}

In this section, we will identify the relevant elements of the CNMP framework. The technique of our approach is shown in detail in Fig. 1.

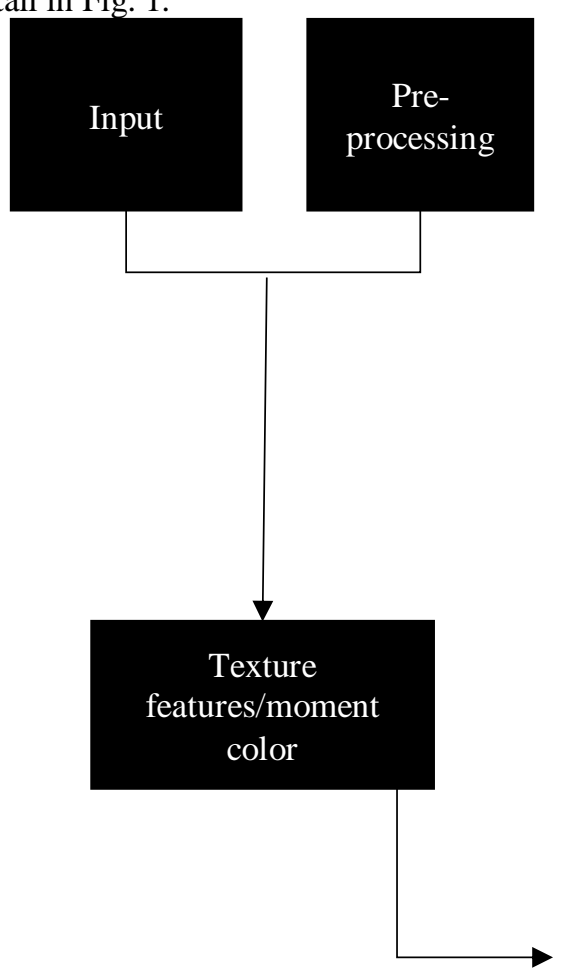

Fusion of features
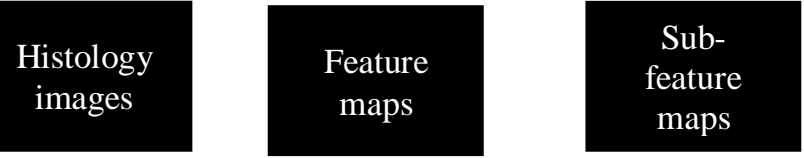

High-level

features

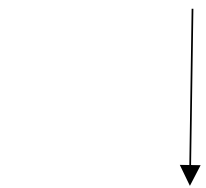

Extraction of highlevel features

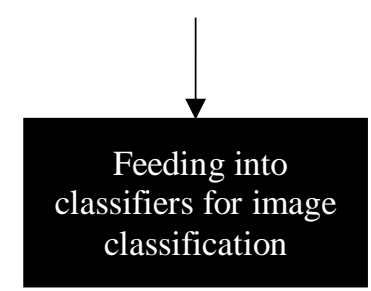

Fig 1. The strategic model

The Convolutional Neural Networks (CNNs) have been extensively utilized in image analysis, video identification, and object recognition following the advent of LeNet-5 in the 90s, and it has achieved great performance in these fields. Convolution layer, pooling layer, one or more completely linked layers, and the softmax layers are often seen in CNNs. For feature extraction, convolutional layers are paired with pooling layer. The classifiers are referred to as the softmax layer. The following are the deep model's primary design fundamentals: (1) to conduct picture processing, e.g. eliminating the mean RGB values and depigmentation of ZCA; (2) to select an effective activation functions; and (3) to determine the starting weights. The deep network will not be capable of learning if the starting weights are too minimal, and if they are too high, the initial weights will diverge. (4) data augmentations, egg extracting random patches from the initial clinical images and flipping clinical images, which are fundamental in biomedical image evaluation; (5) employing dropout to reduce local and overfitting response normalization to minimize the rate of errors are both fundamental; and (6) choosing an effective learning rate. The most typical practice is for the learning rate to decline with each epoch; the most crucial concept is (7) deep networks system. This is endorsed by the facts, which they earned a sophisticated result on ILSVRC 2014 and ILSVRC 2013 correspondingly.

\section{ANALYSIS}

We used MatConvnet, a Matlab package that creates convolution neural network, to design the coding network to retrieve high-level elements, and the conventional datasets centred on colour moment, texture features. On two benchmark clinical image data, we developed a series of tests to validate the usefulness of our technique. The HIS2828 dataset is one, while the 
ISIC2017 dataset is the other. All of our tests were carried out on a machine with an i5-6500 $3.2 \mathrm{GHz}$ processor, a GTX1060 GPU, and 32 GB of system memory.

\section{ISI2017 and HIS2828 Datasets}

The HIS2828 database is made up of four distinct kinds of basic tissue images that comprise multiple types of tissue. Each picture is a 720 by 480-pixel RGB image. The following is a list of the 2828 photos in this dataset: We used 1, 2, 3, and 4 to symbolize the tags in 1026 nerve tissue photos, 484 collagenous images, 804 squamous epithelial illustrations, and 514 muscle tissue illustrations. The HIS2828 dataset's composition is shown in Table 3.

Table 1. HIS-2828 dataset composition

\begin{tabular}{lll}
\hline Image Classification & No. of images & Labels \\
\hline Nervous tissues & 1,026 & 1 \\
Connective tissues & 484 & 2 \\
Epithelial tissues & 804 & 3 \\
Muscular tissues & 514 & 4 \\
\hline
\end{tabular}

The ISIC2017 (International Skin Imaging Collaboration 2017) has has produced a dataset of skin infections. There are 2000 photos in all, 374 of which are dangerous skin cancers called "melanoma" and approximately 1626 that are mild skin cancers called "seborrheic keratosis nevus." Resultantly, it is a binary image evaluation issue, which separates (a) seborrheic keratosis nevus; (b) melanoma. We must deal with the fact that each picture in this collection has a different resolution. The ISIC2017 dataset's composition is shown in Table 4.

Table 2. ISIC-2017 dataset composition

\begin{tabular}{lll}
\hline Image classification & No. of images & Labels \\
\hline Melanoma & 374 & 1 \\
Seborreic keratosis Nevuls & 1,626 & 2 \\
\hline
\end{tabular}

We used the following setup to assess our experiments. To begin, every data was separated into three distinct segments; labeled training, and testing datasets, with a ration of 7:1:2. Therefore, employing a ten-fold cross authentication, all of the approaches were assessed. The photos were then trimmed at randomized from the entire dataset to create fixed-size 140 by 140 images for input into the coding networks. Each picture in the HIS 2828 data was arbitrarily trimmed to $420 \times 420$ pixels before being enlarged to the fixed size 140 by 140 image. Prior to scaling to $140 \times 140$ for the ISIC2017 data, we identified randomized patch with two-thirds of the initial width and length for photos of various sizes. This would save a significant amount of picture data while also reducing processing complexity. These works may be used to create not just fixed-size pictures, but also to enhance image samples. We'd also flip the picture horizontally or vertically to enhance the image datasets even further. The network produces a forecast for every patch at test time, as well as an average of the softmax layer's forecasts if the patch are from the same picture. In the following studies, the effect of image augmenting on precision and runtime will be addressed.

Table 1 shows the network topology of our source code system in detail. It may convergence after 45 epoches, as demonstrated in Table 1. Finally, we employed ReLus as an input signal for convolution layers. Aside from that, batch normalization was used to speed up deep network learning.

\section{Precision}

In this part, we'll perform a series of tests on two genuine medical picture datasets to see how accurate they are and how long they take to execute algorithms. The proportion of properly categorized medical photos is what we're talking about when we say accuracy. Receiver Operating Characteristic (ROC) and confusion matrix curve are utilized to evaluate the framework to effectively compare the methodologies. In the evaluation of the multi-category image classification approach, the confusion matrix represents a table format, which could show a false negative, true negative, false negative and true positive rate. ROC curve represents a visual representation established with a comparison of the TPR (True Positive Rate) to FPR (False Positive Rate) using distinct parameters, whereby FPR and TPR are illustrated: $T P R=\frac{T P}{F N^{\prime}}+T P \ldots \ldots F P R=\frac{F P}{T N^{\prime}}+$ FP where TP, FN, FP, TN represent true positive, false negative, false positive and true negative, respectively.

The ability to demonstrate the classifier image analysis algorithm's effectiveness is quite beneficial. Before the image of the DL model, the Support Vector Machine (SVM) was decisive to for unified classification algorithms in DL; therefore, conventional features and deep features, which would be the combination of conventional and deep attributes, will be contrasted to the CNMP framework. We use the LibSVM-3.17 module to train a Radial Basis Function (RBF) kernal onevs-one classifier. A contrast with the coded networks is important to illustrate the usefulness of integrating characteristics. In addition, the CNMP incorporates a superior feature fusion strategy than R data augmentation and KPCA feature fusion. Because it could trace the features into chaotic space, we used KPCA with the RBF kernal to fusing elements. To accomplish the categorization operation, the feature matching vectors will be sent into Softmax. 
Table 5 displays the accuracy findings of the HIS2828 and ISIC2017 datasets. On both datasets, our technique has the highest accuracy rate of 90.2 and 90.1. The accuracy of SVM (a conventional feature) is the least accurate, as seen in the table. We may get better results even if we only used the coding networks to categorize the medical picture. This research demonstrates that high-level characteristics may better reflect a medical picture than standard features. Our model is more accurate than the two previous ways, thus merging the two kinds of features may be beneficial since the integrated features will potentially signify the images from a multi-scale viewpoint. SVM is clearly superior than coding networks and SVM (conventional features). Furthermore, when we compare our model to R data augmentation and KPCA feature fusion, we might potentially visualize that automated features do not just attain greater outcomes, but also eliminates the timeconsuming procedure of manually modifying the variables.

Table 3. Comparisons of accuracy for image classification algorithms

\begin{tabular}{llll}
\hline Algorithms & HIS2828 (\%) & ISIC2017 (\%) \\
\hline $\begin{array}{l}\text { Support } \quad \text { Vector } \\
\text { (contemporary features) }\end{array}$ & Machines & 72 & 66 \\
$\begin{array}{l}\text { Coding Networks } \\
\text { CNMP }\end{array}$ & 80 & 75 \\
R-feature fusions & 90 & 90 \\
$\begin{array}{l}\text { Support Vector } \quad \text { Machines } \\
\text { (contemporary and deep features) }\end{array}$ & 86 & 89 \\
KPCA feature fusions & 85 & 78 \\
\hline
\end{tabular}

Accuracy rate, as is widely known, cannot be utilized to assess an image categorization system, especially whenever the image data has an uneven dispersion. The HIS2018 database clearly has a sampling imbalances issue. In this case, we use the error matrix to examine the techniques in order to make a more accurate comparison. The first 4 diagonal elements in a confusion matrix reflect the amount and proportion of accurate predictions generated by algorithms on the test datasets. Pink-shading cell signifies inaccurate prediction, and the proportions signifies an overall data amount in the test's datasets The gray columns in the final matrix column represent the recollections and rate of sensitivity of each class while the gray column in the last row alludes to the accuracy rates for every class. Lastly, the total accuracy is represented by the last orthogonal cell. Fig. 2 shows that since neural and epithelium tissues have much more training data, they may achieve greater accuracy and recollection.

Furthermore, as shown in Figure 2d, the CNMP approach has the greatest accuracy and recalls in all categories, demonstrating the effectiveness of our approach. With the oddity of the other 2 groups, $\mathrm{R}$ feature fusion does have the $2^{\text {nd }}$ greatest effectiveness, which is comparable to CNMP in nerve fibers and epithelial; Figures $2 \mathrm{a}$ and $2 \mathrm{~b}$ demonstrate that the source code connectivity can achieve a better result than SVM (conventional features). Nevertheless, the SVM (conventional features) and coder network are hampered by the unemployed multilevel characteristics. Furthermore, Figure 2a shows that the SVM (conventional features) is most susceptible to the fluctuation. If it straightforwardly concatenates the attributes, as shown in Figures $2 \mathrm{c}$ and $2 \mathrm{e}$, it is very poor practice. Figure $2 \mathrm{f}$ vs. Figure $2 \mathrm{~d}$ shows the efficacy of our fusion approach once more. Whenever an image database has an imbalance issue, the SVM (conventional features) might have poor classification efficiency. Rather, the deep prototype might be best at detecting this issue and producing a positive improvement. 


\begin{tabular}{|c|c|c|c|c|c|c|}
\hline \multirow{6}{*}{ Output category } & 4 & $\begin{array}{c}198 \\
11.7 \%\end{array}$ & $\begin{array}{c}26 \\
1.5 \%\end{array}$ & $\begin{array}{c}34 \\
2.0 \%\end{array}$ & $\begin{array}{c}25 \\
1.5 \%\end{array}$ & $\begin{array}{l}70.0 \% \\
30.0 \%\end{array}$ \\
\hline & 3 & $\begin{array}{c}37 \\
2.2 \%\end{array}$ & $\begin{array}{c}388 \\
22.9 \%\end{array}$ & $\begin{array}{c}33 \\
1.9 \%\end{array}$ & $\begin{array}{c}65 \\
3.8 \%\end{array}$ & $\begin{array}{l}74.2 \% \\
25.8 \%\end{array}$ \\
\hline & 3 & $\begin{array}{c}18 \\
1.1 \%\end{array}$ & $\begin{array}{c}21 \\
1.2 \%\end{array}$ & $\begin{array}{c}164 \\
9.7 \%\end{array}$ & $\begin{array}{c}30 \\
1.8 \%\end{array}$ & $\begin{array}{l}70.4 \% \\
29.6 \%\end{array}$ \\
\hline & 4 & $\begin{array}{c}47 \\
2.8 \%\end{array}$ & $\begin{array}{c}59 \\
3.5 \%\end{array}$ & $\begin{array}{c}77 \\
4.5 \%\end{array}$ & $\begin{array}{c}474 \\
27.9 \%\end{array}$ & $\begin{array}{l}72.1 \% \\
27.9 \%\end{array}$ \\
\hline & & $\begin{array}{l}66.0 \% \\
34.0 \%\end{array}$ & $\begin{array}{l}78.5 \% \\
21.5 \%\end{array}$ & $\begin{array}{l}53.2 \% \\
46.8 \%\end{array}$ & $\begin{array}{l}79.8 \% \\
20.2 \%\end{array}$ & $\begin{array}{l}72.2 \% \\
27.8 \%\end{array}$ \\
\hline & & 1 & 2 & 3 & 4 & \\
\hline
\end{tabular}

Fig 2a. Comparisons of the error matrix on the histology data: SVM error matrix (conventional features)

\begin{tabular}{|c|c|c|c|c|c|c|}
\hline \multirow{6}{*}{ Output category } & 4 & $\begin{array}{c}211 \\
12.4 \%\end{array}$ & $\begin{array}{c}20 \\
1.2 \%\end{array}$ & $\begin{array}{c}19 \\
1.1 \%\end{array}$ & $\begin{array}{c}21 \\
1.2 \%\end{array}$ & $\begin{array}{l}77.9 \% \\
22.1 \%\end{array}$ \\
\hline & 3 & $\begin{array}{c}30 \\
1.8 \%\end{array}$ & $\begin{array}{c}394 \\
23.2 \%\end{array}$ & $\begin{array}{c}35 \\
2.1 \%\end{array}$ & $\begin{array}{c}42 \\
2.5 \%\end{array}$ & $\begin{array}{l}78.6 \% \\
21.4 \%\end{array}$ \\
\hline & 3 & $\begin{array}{c}25 \\
1.5 \%\end{array}$ & $\begin{array}{c}28 \\
1.7 \%\end{array}$ & $\begin{array}{c}241 \\
14.2 \%\end{array}$ & $\begin{array}{c}27 \\
1.6 \%\end{array}$ & $\begin{array}{l}75.1 \% \\
24.9 \%\end{array}$ \\
\hline & 4 & $\begin{array}{c}29 \\
1.7 \%\end{array}$ & $\begin{array}{c}59 \\
3.5 \%\end{array}$ & $\begin{array}{c}18 \\
1.1 \%\end{array}$ & $\begin{array}{c}497 \\
29.3 \%\end{array}$ & $\begin{array}{l}82.4 \% \\
17.6 \%\end{array}$ \\
\hline & & $\begin{array}{l}71.5 \% \\
28.5 \%\end{array}$ & $\begin{array}{l}78.6 \% \\
21.4 \%\end{array}$ & $\begin{array}{l}77.0 \% \\
23.0 \%\end{array}$ & $\begin{array}{l}84.7 \% \\
15.3 \%\end{array}$ & $\begin{array}{l}79.2 \% \\
20.8 \%\end{array}$ \\
\hline & & 1 & 2 & 3 & 4 & \\
\hline
\end{tabular}

Fig 2b. Comparisons of the error matrix on the histology data: Error matrix of coded networks 


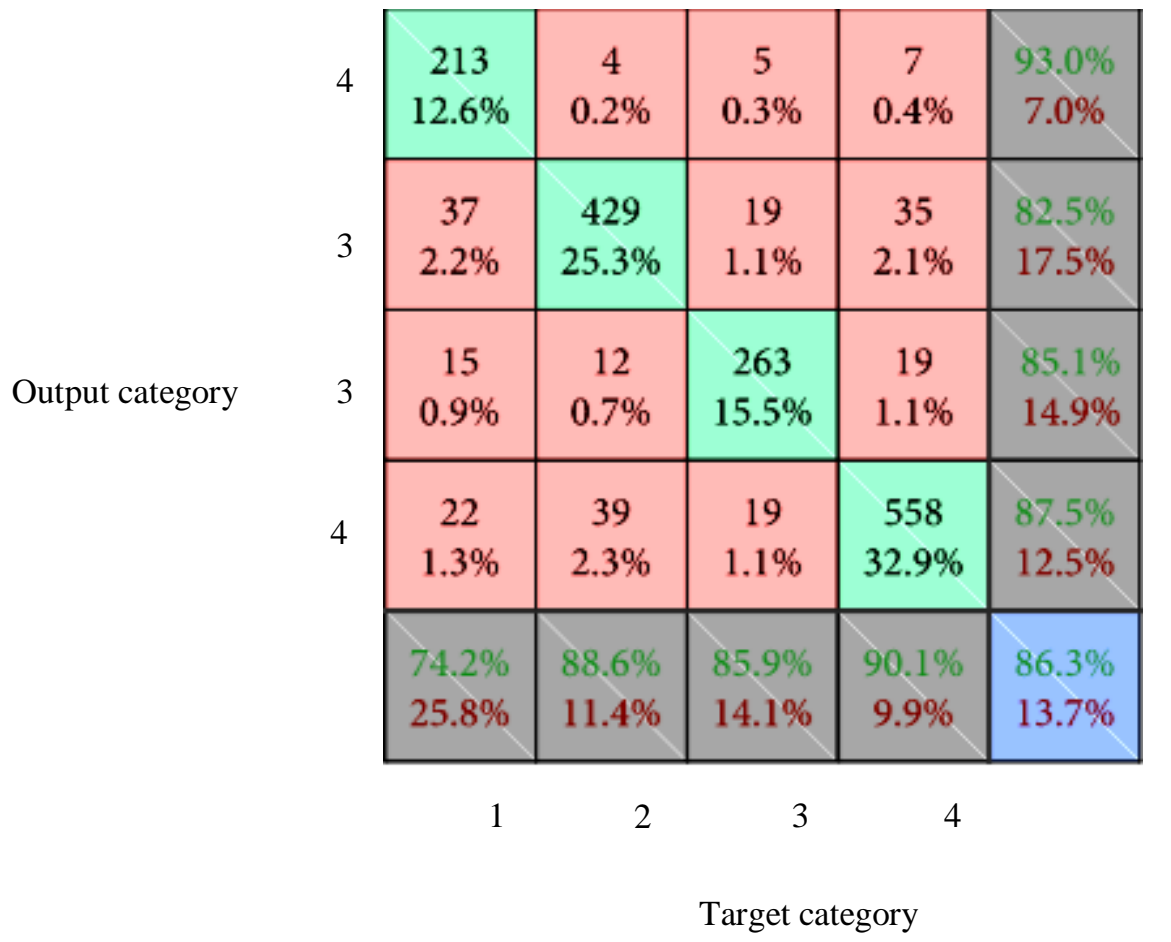

Fig 2c. Comparisons of the error matrix on the histology data: Error matrix of $\mathrm{R}$ feature matching

\begin{tabular}{|c|c|c|c|c|c|c|}
\hline \multirow{6}{*}{ Output category } & 4 & $\begin{array}{c}247 \\
14.6 \%\end{array}$ & $\begin{array}{c}11 \\
0.6 \%\end{array}$ & $\begin{array}{c}7 \\
0.4 \%\end{array}$ & $\begin{array}{c}12 \\
0.7 \%\end{array}$ & $\begin{array}{l}89.2 \% \\
10.8 \%\end{array}$ \\
\hline & 3 & $\begin{array}{c}19 \\
1.1 \%\end{array}$ & $\begin{array}{c}408 \\
24.1 \%\end{array}$ & $\begin{array}{c}5 \\
0.3 \%\end{array}$ & $\begin{array}{c}26 \\
1.5 \%\end{array}$ & $\begin{array}{l}89.1 \% \\
10.9 \%\end{array}$ \\
\hline & 3 & $\begin{array}{c}13 \\
0.8 \%\end{array}$ & $\begin{array}{c}11 \\
0.6 \%\end{array}$ & $\begin{array}{c}284 \\
16.7 \%\end{array}$ & $\begin{array}{c}12 \\
0.7 \%\end{array}$ & $\begin{array}{l}88.8 \% \\
11.2 \%\end{array}$ \\
\hline & 4 & $\begin{array}{c}11 \\
0.6 \%\end{array}$ & $\begin{array}{c}28 \\
1.7 \%\end{array}$ & $\begin{array}{c}12 \\
0.7 \%\end{array}$ & $\begin{array}{c}590 \\
34.8 \%\end{array}$ & $\begin{array}{c}92.0 \% \\
8.0 \%\end{array}$ \\
\hline & & $\begin{array}{l}85.2 \% \\
14.8 \%\end{array}$ & $\begin{array}{l}89.1 \% \\
10.9 \%\end{array}$ & $\begin{array}{c}92.2 \% \\
7.8 \%\end{array}$ & $\begin{array}{c}92.2 \% \\
7.8 \%\end{array}$ & $\begin{array}{c}90.2 \% \\
9.8 \%\end{array}$ \\
\hline & & 1 & 2 & 3 & 4 & \\
\hline
\end{tabular}

Fig 2d. Comparisons of the error matrix on the histology data: Error matrix of CNMP 


\begin{tabular}{|c|c|c|c|c|c|c|}
\hline \multirow{5}{*}{ Output category } & 4 & $\begin{array}{c}232 \\
13.7 \%\end{array}$ & $\begin{array}{c}15 \\
0.9 \%\end{array}$ & $\begin{array}{c}23 \\
1.4 \%\end{array}$ & $\begin{array}{c}14 \\
0.8 \%\end{array}$ & $\begin{array}{l}81.7 \% \\
18.3 \%\end{array}$ \\
\hline & 3 & $\begin{array}{c}17 \\
1.0 \%\end{array}$ & $\begin{array}{c}384 \\
22.6 \%\end{array}$ & $\begin{array}{c}18 \\
1.1 \%\end{array}$ & $\begin{array}{c}41 \\
2.4 \%\end{array}$ & $\begin{array}{l}83.5 \% \\
16.5 \%\end{array}$ \\
\hline & 3 & $\begin{array}{c}34 \\
2.0 \%\end{array}$ & $\begin{array}{c}10 \\
0.6 \%\end{array}$ & $\begin{array}{c}211 \\
12.4 \%\end{array}$ & $\begin{array}{c}18 \\
1.1 \%\end{array}$ & $\begin{array}{l}77.3 \% \\
22.7 \%\end{array}$ \\
\hline & 4 & $\begin{array}{c}34 \\
2.0 \%\end{array}$ & $\begin{array}{c}52 \\
3.1 \%\end{array}$ & $\begin{array}{c}45 \\
2.7 \%\end{array}$ & $\begin{array}{c}548 \\
32.3 \%\end{array}$ & $\begin{array}{l}80.7 \% \\
19.3 \%\end{array}$ \\
\hline & & $\begin{array}{l}73.2 \% \\
26.8 \%\end{array}$ & $\begin{array}{l}83.3 \% \\
16.7 \%\end{array}$ & $\begin{array}{l}71.0 \% \\
29.0 \%\end{array}$ & $\begin{array}{l}88.2 \% \\
11.8 \%\end{array}$ & $\begin{array}{l}81.1 \% \\
18.9 \%\end{array}$ \\
\hline
\end{tabular}

Target category

Fig 2e. Comparisons of the error matrix on the histology data: Error matrix of SVMs (conventional and deep features)

\begin{tabular}{|c|c|c|c|c|c|c|}
\hline \multirow{6}{*}{ Output category } & 4 & $\begin{array}{c}254 \\
15.0 \%\end{array}$ & $\begin{array}{c}14 \\
0.8 \%\end{array}$ & $\begin{array}{c}19 \\
1.1 \%\end{array}$ & $\begin{array}{c}13 \\
0.8 \%\end{array}$ & $\begin{array}{l}84.7 \% \\
15.3 \%\end{array}$ \\
\hline & 3 & $\begin{array}{c}12 \\
0.7 \%\end{array}$ & $\begin{array}{c}396 \\
23.3 \%\end{array}$ & $\begin{array}{c}11 \\
0.6 \%\end{array}$ & $\begin{array}{c}33 \\
1.9 \%\end{array}$ & $\begin{array}{l}87.6 \% \\
12.4 \%\end{array}$ \\
\hline & 3 & $\begin{array}{c}24 \\
1.4 \%\end{array}$ & $\begin{array}{c}8 \\
0.5 \%\end{array}$ & $\begin{array}{c}229 \\
13.5 \%\end{array}$ & $\begin{array}{c}14 \\
0.8 \%\end{array}$ & $\begin{array}{l}83.3 \% \\
16.7 \%\end{array}$ \\
\hline & 4 & $\begin{array}{c}27 \\
1.6 \%\end{array}$ & $\begin{array}{c}43 \\
2.5 \%\end{array}$ & $\begin{array}{c}38 \\
2.2 \%\end{array}$ & $\begin{array}{c}561 \\
33.1 \%\end{array}$ & $\begin{array}{l}83.9 \% \\
16.1 \%\end{array}$ \\
\hline & & $\begin{array}{l}80.1 \% \\
19.9 \%\end{array}$ & $\begin{array}{l}85.9 \% \\
14.1 \%\end{array}$ & $\begin{array}{l}77.1 \% \\
22.9 \%\end{array}$ & $\begin{array}{c}90.3 \% \\
9.7 \%\end{array}$ & $\begin{array}{l}84.9 \% \\
15.1 \%\end{array}$ \\
\hline & & 1 & 2 & 3 & 4 & \\
\hline
\end{tabular}

Fig 2f. Comparisons of the error matrix on the histology data: Error matrix of KPCA feature matching

Fig. 2: Confusion matrix contrast on the histopathology dataset; (a) SVM error matrix (conventional features); (b) coding connectivity confusion error matrix; (c) R feature matching confusion error matrix; (d) CNMP error matrix; (e) SVM error matrix (conventional and complex feature); (f) KPCA feature matching error matrix. The Receiver Operating Characteristics (ROCs) curve signifies a visual representation of FPR and TPR retrieved from distinct thresholds. Since the binary data integrates a sample unbalanced problem, evaluating the rate of performance of a two-class image clustering technique is relevant.

\section{CONCLUSION}

We introduce a novel clinical image classification system known as CNMP that blends high-level feature extraction from coded networks with standard image characteristics. To our knowledge, this is the first instance a deep algorithm has been 
used to categorize medical pictures directly using standard image attributes. On the ISIC2017 and HIS2828 image datasets, our solution achieves a percentage precision of 90.2 and 90.1, respectively; outperforming SVM (conventional features), coded networks, and $\mathrm{R}$ feature fusion by a wide margin. We also explore how picture expansion affects the algorithm's precision and execution time. Future research might include using an effective pruning approach to drastically decrease the constraints. Furthermore, in the future, we may use "Network in Network" (NIN) to get stronger non-linear high-level attributes for reconstructions of medical pictures, which may outperform our model. We're interested in creating further feature fusion solutions, such as Multi-Feature Fusion Deep Network (MFFDN) based on deblurring auto-encoders or metaspace blending to merge homogenous models.

\section{References}

[1] G. Tian et al., "Adding before pruning: Sparse filter fusion for deep convolutional neural networks via auxiliary attention," IEEE Trans. Neural Netw. Learn. Syst., vol. PP, 2021.

[2] T. Pladere, M. Velina, V. Andriksone, R. Pitura, K. Panke, and G. Krumina, "Visual search in three-dimensional non-medical images: visualmotor performance of radiologists," in Fourth International Conference on Applications of Optics and Photonics, 2019.

[3] J. Yuan, A.-T. Chiang, W. Tang, and A. Haro, "EProduct: A million-scale visual search benchmark to address product recognition challenges," arXiv [cs.CV], 2021.

[4] O. Kunickaya et al., "Using machine vision to improve the efficiency of lumber mills," J. Phys. Conf. Ser., vol. 1478, no. 1, p. 012020, 2020.

[5] J. Metan, A. Y. Prasad, K. S. Ananda Kumar, M. Mathapati, and K. K. Patil, "Cardiovascular MRI image analysis by using the bio inspired (sand piper optimized) fully deep convolutional network (Bio-FDCN) architecture for an automated detection of cardiac disorders," Biomed. Signal Process. Control, vol. 70, no. 103002, p. 103002, 2021.

[6] A. Bedoui and M. Et-tolba, "A deep neural network-based interference mitigation for MIMO-FBMC/OQAM systems," Front. Comms. Net., vol. 2, 2021.

[7] J. Gu, Y. Lu, and G. Xu, "Mismatched lesions on 18F-FDG PET and 18F-fluciclovine PET images in a patient with metastatic prostate small cell carcinoma," Clin. Nucl. Med., vol. Publish Ahead of Print, 2021.

[8] M. W. Lafarge and V. H. Koelzer, "Rotation invariance and extensive data augmentation: A strategy for the mitosis domain generalization (MIDOG) challenge," arXiv [cs.CV], 2021.

[9] S. Pang, Z. Yu, and M. A. Orgun, “A novel end-to-end classifier using domain transferred deep convolutional neural networks for biomedical images," Comput. Methods Programs Biomed., vol. 140, pp. 283-293, 2017.

[10] M. Liu, L. Chen, X. Du, L. Jin, and M. Shang, “Activated gradients for deep neural networks," IEEE Trans. Neural Netw. Learn. Syst., vol. PP, pp. 1-13, 2021.

[11] G. Son and Y. Kim, "EEG-based emotion classification for verifying the Korean emotional movie clips with Support Vector Machine (SVM)," Complexity, vol. 2021, pp. 1-14, 2021

[12] O. Reiter et al., "The differences in clinical and dermoscopic features between in situ and invasive nevus-associated melanomas and de novo melanomas," J. Eur. Acad. Dermatol. Venereol., vol. 35, no. 5, pp. 1111-1118, 2021.

[13] A. B. Beasley et al., "Low-Pass whole-genome sequencing as a method of determining copy number variations in uveal melanoma tissue samples," J. Mol. Diagn., vol. 22, no. 3, pp. 429-434, 2020.

[14] D. Pandiar, S. Basheer, P. M. Shameena, S. Sudha, and L. J. Dhana, “Amelanotic melanoma masquerading as a granular cell lesion,” Case Rep. Dent., vol. 2013, p. 924573, 2013.

[15] H. Chegraoui et al., "Object detection improves tumour segmentation in MR images of rare brain tumours," Cancers (Basel), vol. 13, no. 23, p. $6113,2021$.

[16] D. S. Abou et al., "Preclinical single photon emission computed tomography of alpha particle-emitting radium-223," Cancer Biother. Radiopharm., vol. 35, no. 7, pp. 520-529, 2020.

[17] C. M. Rumack and M. L. Johnson, "Role of computed tomography and ultrasound in neonatal brain imaging," J. Comput. Tomogr., vol. 7, no. 1, pp. 17-29, 1983.

[18] J.-Y. Li, Z.-H. Zhan, J. Xu, S. Kwong, and J. Zhang, "Surrogate-assisted hybrid-model estimation of distribution algorithm for mixed-variable hyperparameters optimization in convolutional neural networks," IEEE Trans. Neural Netw. Learn. Syst., vol. PP, pp. 1-15, 2021.

[19] T. Song, L. Xin, C. Gao, T. Zhang, and Y. Huang, "Quaternionic extended local binary pattern with adaptive structural pyramid pooling for color image representation,” Pattern Recognit., vol. 115, no. 107891, p. 107891, 2021.

[20] X. Peng, X. Gao, and X. Li, “On better training the infinite restricted Boltzmann machines," Mach. Learn., vol. 107, no. 6, pp. 943-968, 2018.

[21] A. G. Nagesha, G. Mahesh, and N. A. Gowrishankar, "Identifying DDoS attacks in 4G networks using artificial neural networks and principal component analysis," Int. j. netw. virtual organ., vol. 25, no. 1, p. 14, 2021.

[22] G. Rajesh and A. Chaturvedi, "Data reconstruction in heterogeneous environmental wireless sensor networks using robust tensor principal component analysis,” IEEE Trans. Signal Inf. Process. Netw., vol. 7, pp. 539-550, 2021.

[23] P. Chen, C. Agarwal, and A. Nguyen, "The shape and simplicity biases of adversarially robust ImageNet-trained CNNs," arXiv [cs.CV], 2020.

[24] E. Bellon et al., "PACS/HIS integration in handling and viewing ICU images generated by a phosphorplate scanner," in Medical Imaging 1996: PACS Design and Evaluation: Engineering and Clinical Issues, 1996.

[25] S. E. Zirka, Y. I. Moroz, and C. M. Arturi, "Once again about the indivisibility of the scattered inductance of the transformer," Electr. Eng. Power Eng., no. 1, pp. 8-17, 2021.

[26] A. Rampun, B. Scotney, P. Morrow, H. Wang, and J. Winder, "Breast density classification using local quinary patterns with various neighbourhood topologies," J. Imaging, vol. 4, no. 1, p. 14, 2018.

[27] F. Avau, M. Chintinne, S. Baudry, and F. Buxant, "Literature review and case report of bilateral intracystic papillary carcinoma associated with an invasive ductal carcinoma in a male breast," Breast Dis., vol. 41, no. 1, pp. 5-13, 2022. 
[28] E. Ahn, A. Kumar, J. Kim, C. Li, D. Feng, and M. Fulham, "X-ray image classification using domain transferred convolutional neural networks and local sparse spatial pyramid," in 2016 IEEE 13th International Symposium on Biomedical Imaging (ISBI), 2016.

[29] S. A. Rahim and G. Manson, "Kernel principal component analysis for structural health monitoring and damage detection of an engineering structure under operational loading variations," J. Fail. Anal. Prev., 2021.

[30] A. Ali, Y. Zhu, and M. Zakarya, "Exploiting dynamic spatio-temporal graph convolutional neural networks for citywide traffic flows prediction," Neural Netw., vol. 145, pp. 233-247, 2022. 